\title{
Frequency aware robust economic dispatch
}

\author{
Donglei SUN ${ }^{1}$, Xueshan HAN ${ }^{1}$, Bo ZHANG ${ }^{1}$, Mingqiang WANG ${ }^{1}$, \\ Tianyu DING ${ }^{2}$
}

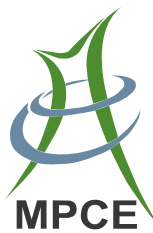

\begin{abstract}
This paper proposes a novel frequency aware robust economic dispatch (FARED) approach to exploit the synergistic capability of accommodating uncertain loads and renewable generation by accounting for both the frequency regulation effect and optimal participation mechanism of secondary regulation reserves for conventional units in response to uncertainties in the robust optimization counterpart of security constrained economic dispatch. The FARED is formulated as a robust optimization problem. In this formulation the allowable frequency deviation and the possible load or renewable generation curtailments are expressed in terms of variable uncertainty sets. The variables in the formulation are described as interval variables and treated in affine form. In order to improve the computational tractability, the dominant constraints which can
\end{abstract}

CrossCheck date: 1 September 2015

Received: 5 March 2015/Accepted: 1 September 2015/Published online: 18 February 2016

(C) The Author(s) 2016. This article is published with open access at Springerlink.com

$\triangle$ Donglei SUN

sdusdlei@sina.com

Xueshan HAN

xshan@sdu.edu.cn

Bo ZHANG

zb@sdu.edu.cn

Mingqiang WANG

wang0367@sdu.edu.cn

Tianyu DING

452604208@qq.com

1 Key Laboratory of Power System Intelligent Dispatch and Control of Ministry of Education, Shandong University, Jinan 250061, China

2 Department of Electrical and Computer Engineering, University of Alberta, Edmonton, AB T6G 2G7, Canada be the candidates of tight transmission constraints are determined by complementarity constraints. Then the robust optimization problem is simplified to a bilinear programming problem based on duality theory. Finally, the effectiveness and efficiency of the proposed method are illustrated based on several study cases.

Keywords Economic dispatch, Frequency regulation effect, Interval uncertainty, Robust optimization, Affine policy, Complementarity constraints

\section{Introduction}

The study of economic dispatch dates back to the 1920s. The spatial and temporal distribution variation of load demand is the prime cause of economic dispatch. The goal of dispatch is the advanced decision-making for base-point generations of units and configuration of spinning reserves based on load forecast. From classical economic dispatch centering on the concepts of incremental cost, incremental loss and hydro-coal conversion equivalent in the mid and late of 1950s [1], to the optimal power flow considering network security in the 1960s [2], thence to the theories of dispatch and control which emphasizing the coordination of dispatch and automatic generation control (AGC) in the 1980s [3, 4], the study of economic dispatch has been relatively mature in theory and practice.

However, with the evolution of electrical loads and the large-scale integration of volatile renewable generations, power system dispatch and operation faces the challenge of increasing injection uncertainties [5]. Injection uncertainties lead to the uncertainties of branch flow, which poses great threats to network security. Security constrained economic dispatch under uncertainty is urgent to address. 
In the context of power schedule under uncertainty, many approaches have been developed. Some use advanced mathematical techniques to model the uncertainties, including probabilistic distribution [6, 7], fuzzy arithmetic [8,9] and interval arithmetic [10, 11]. The former two descriptions require the knowledge of membership functions or probability distribution whereas the uncertainties can be easily described by interval arithmetic with upper and lower bounds, which coincides with the available load or wind power forecast methods $[12,13]$. Furthermore, the use of interval arithmetic is commensurate with the available robust optimization techniques $[14,15]$. Some deal with uncertainties through surrogate constraints, e.g., ramp requirement $[16,17]$, reserve requirement $[18$, 19], network security constraints [20, 21], and frequency control adequacy requirement [22-24]. Frequency control adequacy requirement means that the deployment of frequency control reserves for the dynamic process to survive the transient period and avoid activation of under frequency load shedding or generator tripping subsequent to uncertainties [22-24] or the postulated disturbances [2527]. Frequency control adequacy is ensured by adding frequency constraints using the simplified model of frequency dynamics or through deployment of reserves based on practice experience. Others exploit the potential capability of power system to address uncertainties. Reference [28] utilizes the short time overloading capability of generating units to promote the accommodation of uncertainties on the decision-making level. Reference [29] accounts for the response mechanism of system reserves to identify the maximum renewable generation ranges that power system can accommodate. Reference [21] introduces the optimal response mechanism of secondary reserves in response to uncertainties based on steady-state security region method. However, the system frequency is usually assumed to remain nominal implicitly in normal mode, or the frequency deviation from nominal in contingency mode is limited to be the supplementary constraint in the dispatch decision process. This assumption is more restrictive than in reality, for the frequency variation within a certain range is allowable in the normal system operation. Thus the decision results are rather conservative especially when the uncertainties are considered.

The key to power schedule under uncertainty is to determine the coordination strategies of dispatch and control while satisfying the security constraints. Reference [30] presents a model-based joint economic dispatch and frequency control decision scheme to tackle the variation of intermittent resources, but without the model of uncertainties. In [19], a synergistic economic dispatch concerning the frequency regulation effect is proposed, but the units' response mechanism to uncertainties is not involved. In $[19,30]$, the frequency deviation is incorporated explicitly into the economic decision process, which is instructive for the potential economic and technical benefits of accommodating uncertainties. However, the network model is not well considered. It has received hardly any attention simultaneous accounting for the frequency regulation effect and the response mechanism of secondary reserve of AGC units together with network constraints for power schedule problem. Therefore, this paper proposes a frequency aware robust economic dispatch (FARED) to accommodate uncertainties by accounting for both the frequency regulation effect and the unit reserve response mechanism in the robust optimization counterpart of traditional security constrained economic dispatch.

The contributions of this paper are highlighted as follows.

1) The frequency regulation effect and the unit reserve response mechanism are considered in economic dispatch. The allowable frequency deviation and participation factors for units are included into FARED, and this approach can exploit the synergistic capability on the decisionmaking level to accommodate the uncertain loads and renewable generations.

2) With the introduction of affine policy, a robust optimization formulation with the variable uncertainty sets for FARED is proposed. It can provide the system operators with the robust solution that immunizes all possible realizations under interval uncertainties.

3) Dominant constraints which can be the candidates of tight transmission constraints are determined based on complementarity constraints, then the model can be well simplified and the computation tractability is improved.

The rest of the paper is organized as follows. Section 2 describes the definition and classification of synergistic capability of power system. In Section 3, the mathematical model of FARED is described. Section 4 gives the solution methodology. In Section 5, numerical studies are presented to demonstrate validity and efficiency of the proposed method. Section 6 draws the conclusions and discussions.

\section{Definition and classification of synergistic capability in power system}

Sources and sinks have the negative feedback properties to respond to the variation of the system frequency. If the system frequency increases, the output power of sources will reduce while the consumption of sinks will increase. Conversely, if the frequency decreases, the output power of sources will increase while the consumption of sinks will reduce. This is referred to as the frequency regulation effect, and it endows the system with potential capability of automatically balancing the supply and demand under uncertainty. The existence of frequency regulation effect 
facilitates the power system scheduling in terms of reducing the spinning reserve requirements and curtailments of loads and renewable generations on the decision-making level. Therefore, both the generations and loads have the capability to automatically adapt to the power balance under uncertainty, which is the technical foundation for the automatic operation of artificial power system. When the frequency regulation effect is considered for power scheduling, the supply and demand do not need to be matched perfectly under the nominal frequency, thus the solution space of power scheduling is enlarged. The frequency regulation effect can be represented approximately in linear form as

$P=P^{*}+\beta \Delta f$

where $P$ is the actual power of the source or sink; $P^{*}$ is the constant power component which is independent of frequency; $\Delta f$ is the frequency deviation and $\Delta f=\left(f-f_{0}\right) ; f$ is the actual frequency; $f_{0}$ is the nominal frequency; $\beta$ is the frequency characteristic factor; the frequency related power component $\beta \Delta f$ is the automatic synergistic capability.

However, if the range of the injection uncertainties is large enough beyond the automatic synergistic capability, the system's secondary frequency control will be activated. The activation of AGC units, controllable loads and energy storage system used in the secondary frequency control is termed as the controllable synergistic capability for the basis of the automatic synergistic capability. If the uncertainties increase further, the controllable synergistic capability will run out, and the curtailments of loads and renewable generations are inevitable. The curtailments can be termed as the re-controllable synergistic capability.

In this paper, the power scheduling for an isolated power system with wind power integration is considered. The uncertain loads and wind power are described as interval numbers, and treated in affine form. That is

$$
\begin{aligned}
& \left\{\begin{array}{l}
\tilde{P}_{\mathrm{D} j}=P_{\mathrm{D} j}^{0}+\Delta \tilde{P}_{\mathrm{D} j} \\
\Delta \tilde{P}_{\mathrm{D} j} \in\left[-\Delta P_{\mathrm{D} j}, \Delta P_{\mathrm{D} j}\right]
\end{array} \quad \forall j \in N_{\mathrm{D}}\right. \\
& \left\{\begin{array}{l}
\tilde{P}_{\mathrm{W} k}=P_{\mathrm{W} k}^{0}+\Delta \tilde{P}_{\mathrm{W} k} \\
\Delta \tilde{P}_{\mathrm{W} k} \in\left[-\Delta P_{\mathrm{W} k}, \Delta P_{\mathrm{W} k}\right]
\end{array} \quad \forall k \in N_{\mathrm{W}}\right.
\end{aligned}
$$

where the superscript " $\sim$ " is a realization of interval variable; $\tilde{P}_{\mathrm{D} j}$ is the power demand of load $j ; \tilde{P}_{\mathrm{W} k}$ is the wind power of wind farm $k ; P_{\mathrm{D} j}^{0}, P_{\mathrm{W} k}^{0}, \Delta P_{\mathrm{D} j}$ and $\Delta P_{\mathrm{W} k}$ are the predicted center values and the variation range widths of load and wind power, respectively; $\Delta \tilde{P}_{\mathrm{D} j}$ is the power demand deviation from the predicted center value of load $j$; $\Delta \tilde{P}_{\mathrm{W} k}$ is the wind power deviation from the predicted center value of wind farm $k ; N_{\mathrm{D}}$ and $N_{\mathrm{W}}$ are the set of loads and wind farms, respectively.

For the practical scenarios, the realization of power balance can be expressed as

$$
\begin{aligned}
& \sum_{g \in N_{\mathrm{G}}} P_{\mathrm{G} g}^{0}+\sum_{k \in N_{\mathrm{W}}} P_{\mathrm{W} k}^{0}=\sum_{j \in N_{\mathrm{D}}} P_{\mathrm{D} j}^{0} \\
& \beta_{\mathrm{f}} \Delta \tilde{f}+\sum_{g \in N_{\mathrm{G}}} \Delta \tilde{P}_{\mathrm{G} g}^{\mathrm{c}}+\left\{\sum_{j \in N_{\mathrm{D}}} \Delta \tilde{P}_{\mathrm{D} j}^{\mathrm{rc}}-\sum_{k \in N_{\mathrm{W}}} \Delta \tilde{P}_{\mathrm{W} k}^{\mathrm{rc}}\right\} \\
& =\sum_{j \in N_{\mathrm{D}}} \Delta \tilde{P}_{\mathrm{D} j}-\sum_{k \in N_{\mathrm{W}}} \Delta \tilde{P}_{\mathrm{W} k} \\
& \beta_{\mathrm{f}}=\left(\sum_{g \in N_{\mathrm{G}}} R_{\mathrm{G} g}-\sum_{j \in N_{\mathrm{D}}} D_{\mathrm{D} j}\right)
\end{aligned}
$$

where $P_{\mathrm{G} g}^{0}$ is the base-point generation for unit $g ; N_{\mathrm{G}}$ is the set of conventional units; $\beta_{\mathrm{f}}$ is the system frequency bias factor $[1] ; R_{\mathrm{G}}$ and $D_{\mathrm{D}}$ are the frequency characteristic coefficients of the generation and load, respectively; $\Delta \tilde{f}$, $\Delta \tilde{P}_{\mathrm{G} g}^{\mathrm{c}}, \Delta \tilde{P}_{\mathrm{D} j}^{\mathrm{rc}}$ and $\Delta \tilde{P}_{\mathrm{W} k}^{\mathrm{rc}}$ are the frequency deviation, realization of secondary reserve of AGC units (all units are assumed to be AGC units), curtailments of loads and wind generations, respectively.

Equation (4) corresponds to the base-point power balance. In (5), the first term on the left-hand side corresponds to the automatic synergistic capability, the second term on the left-hand side corresponds to the controllable synergistic capability, and the third term on the left-hand side corresponds to the re-controllable synergistic capability. The left-hand side of (5) represents the total synergistic capability. The right-hand side of (5) represents the total uncertainty in the system. Equation (5) imposes the balance between the synergistic capability and the uncertainties, and it describes the response of synergistic capability in power system operation under uncertainty.

\section{Mathematical model of FARED}

The FARED problem aims at minimizing the operation cost while satisfying all the physical or technical constraints. In this section, the FARED problem is formulated as a robust optimization problem with variable uncertainty sets. For brevity, the overline and underline are used to identify the upper and lower limits of the relevant variables due to physical or technical constraints, and the superscript "max", "min" are the upper and lower bounds of interval variables.

\subsection{Objective function}

The objective function is formulated below.

$$
\begin{gathered}
\min \sum_{g \in N_{\mathrm{G}}}\left\{C_{\mathrm{G} g}^{0}\left(P_{\mathrm{G} g}^{0}\right)+C_{\mathrm{G} g}^{\mathrm{c}}\left(\Delta P_{\mathrm{G} g}^{\mathrm{cup}}, \Delta P_{\mathrm{G} g}^{\mathrm{cdn}}\right)\right\} \\
+\sum_{k \in N_{\mathrm{W}}} C_{\mathrm{W} k}^{\mathrm{rc}}\left(\Delta P_{\mathrm{W} k}^{\mathrm{rc}}\right)+\sum_{j \in N_{\mathrm{D}}} C_{\mathrm{D} j}^{\mathrm{rc}}\left(\Delta P_{\mathrm{D} j}^{\mathrm{rc}}\right)
\end{gathered}
$$


where $C_{\mathrm{G} g}^{0}(\cdot)$ is the production cost function for $g^{\text {th }}$ conventional unit, $C_{\mathrm{G} g}^{0}\left(P_{\mathrm{G} g}^{0}\right)=a_{\mathrm{G} g}\left(P_{\mathrm{G} g}^{0}\right)^{2}+b_{\mathrm{G} g} P_{\mathrm{G} g}^{0}+c_{\mathrm{G} g}$; $a_{\mathrm{G} g}, b_{\mathrm{G} g}, c_{\mathrm{G} g}$ are the cost coefficients; $\Delta P_{\mathrm{G} g}^{\text {cup }}$ and $\Delta P_{\mathrm{G} g}^{\mathrm{cdn}}$ are the upward and downward secondary reserve requirement of $g^{\text {th }}$ unit, respectively; $C_{\mathrm{G} g}^{\mathrm{c}}(\cdot)$ is the reserve cost function, and $C_{\mathrm{G} g}^{\mathrm{c}}\left(\Delta P_{\mathrm{G} g}^{\text {cup }}, \Delta P_{\mathrm{G} g}^{\mathrm{cdn}}\right)=\alpha_{\mathrm{G} g}^{\mathrm{c}} \Delta P_{\mathrm{G} g}^{\mathrm{cup}}-\alpha_{\mathrm{G} g}^{\mathrm{c}} \Delta P_{\mathrm{G} g}^{\mathrm{cdn}}$; the cost coefficient $\alpha_{\mathrm{G} g}^{\mathrm{c}}=2 a_{\mathrm{G} g} \bar{P}_{\mathrm{G} g}+b_{\mathrm{G} g} ; C_{\mathrm{W} k}^{\mathrm{rc}}(\cdot)$ is the cost function for wind power curtailment of $k^{\text {th }}$ wind farm, $C_{\mathrm{W} k}^{\mathrm{rc}}\left(\Delta P_{\mathrm{W} k}^{\mathrm{rc}}\right)=\alpha_{\mathrm{W} k}^{\mathrm{rc}} \Delta P_{\mathrm{W} k}^{\mathrm{rc}} ; \Delta P_{\mathrm{W} k}^{\mathrm{rc}}$ is the wind power curtailment of $k^{\text {th }}$ wind farm; the cost coefficient $\alpha_{\mathrm{W} k}^{\mathrm{rc}}$ is chosen as $\alpha_{\mathrm{WD}}=\max \alpha_{\mathrm{G} g}^{\mathrm{c}}, g \in N_{\mathrm{G}} ; C_{\mathrm{D} j}^{\mathrm{rc}}(\cdot)$ is the cost function for load curtailment of $j^{\text {th }}$ load, $C_{\mathrm{D} j}^{\mathrm{rc}}\left(\Delta P_{\mathrm{D} j}^{\mathrm{rc}}\right)=\alpha_{\mathrm{D} j}^{\mathrm{rc}} \Delta P_{\mathrm{D} j}^{\mathrm{rc}} ; \Delta P_{\mathrm{D} j}^{\mathrm{rc}}$ is the load curtailment of $j^{\text {th }}$ load; the cost coefficient $\alpha_{\mathrm{D} j}^{\text {rc }}$ is set as $\alpha_{\mathrm{WD}}$.

\subsection{Decision variables}

The decision variables include: the base-point generation $P_{\mathrm{G} g}^{0}$ and the participation factor $\beta_{\mathrm{G} g}$ of conventional unit, $g \in N_{\mathrm{G}}$; the upward and downward secondary reserve requirement $\Delta P_{\mathrm{G} g}^{\text {cup }}$ and $\Delta P_{\mathrm{G} g}^{\text {cdn }}$ of conventional unit, $g \in N_{\mathrm{G}}$; the upper and lower system frequency deviation $\Delta f^{\text {up }}$ and $\Delta f^{\mathrm{dn}}$; the load curtailment $\Delta P_{\mathrm{D} j}^{\mathrm{rc}}, j \in N_{\mathrm{D}}$; the wind power curtailment $\Delta P_{\mathrm{W} k}^{\mathrm{rc}}, k \in N_{\mathrm{W}}$.

\subsection{Constraints}

1) Base-point power flow

$\sum_{l \in N_{\mathrm{S}, i}} P_{\mathrm{L} l}^{0}-\sum_{l \in N_{\mathrm{E}, i}} P_{\mathrm{L} l}^{0}=\sum_{g \in N_{\mathrm{G}, i}} P_{\mathrm{G} g}^{0}+\sum_{k \in N_{\mathrm{W}, i}} P_{\mathrm{W} k}^{0}-\sum_{j \in N_{\mathrm{D}, i}} P_{\mathrm{D} j}^{0}$,

$\forall i \in N_{\mathrm{B}}$

where $N_{\mathrm{B}}$ is the set of buses; $N_{\mathrm{G}, i}, N_{\mathrm{W}, i}$, and $N_{\mathrm{D}, i}$ are the sets of units, wind farms, and loads at bus $i$, respectively; $N_{\mathrm{S}, i}$ and $N_{\mathrm{E}, i}$ are the sets of transmission lines with bus $i$ as the "from" bus and the "to" bus, respectively; $P_{\mathrm{L} l}^{0}$ is the power flow on transmission line $l$ for base-point operation pattern.

2) Constraints for synergistic capability requirement

$$
\tilde{P}_{\mathrm{C}}^{\mathrm{max}}=\beta_{\mathrm{f}} \Delta f^{\mathrm{dn}}+\sum_{g \in N_{\mathrm{G}}} \Delta P_{\mathrm{G} g}^{\mathrm{cup}}+\sum_{j \in N_{\mathrm{D}}} \Delta P_{\mathrm{D} j}^{\mathrm{rc} 1}-\sum_{k \in N_{\mathrm{W}}} \Delta P_{\mathrm{W} k}^{\mathrm{rc} 1}
$$

The full coverage of the range of the system uncertainty set should be ensured as

$$
\tilde{P}_{\mathrm{C}}^{\min }=\beta_{\mathrm{f}} \Delta f^{\mathrm{up}}+\sum_{g \in N_{\mathrm{G}}} \Delta P_{\mathrm{G} g}^{\mathrm{cdn}}+\sum_{j \in N_{\mathrm{D}}} \Delta P_{\mathrm{D} j}^{\mathrm{rc} 2}-\sum_{k \in N_{\mathrm{W}}} \Delta P_{\mathrm{W} k}^{\mathrm{rc} 2}
$$

$$
\begin{gathered}
\tilde{P}_{\mathrm{C}}^{\min } \leq \underline{P}_{\mathrm{U}}=-\sum_{j \in N_{\mathrm{D}}} \Delta P_{\mathrm{D} j}-\sum_{k \in N_{\mathrm{W}}} \Delta P_{\mathrm{W} k} \\
\sum_{j \in N_{\mathrm{D}}} \Delta P_{\mathrm{D} j}+\sum_{k \in N_{\mathrm{W}}} \Delta P_{\mathrm{W} k}=\bar{P}_{\mathrm{U}} \leq \tilde{P}_{\mathrm{C}}^{\max }
\end{gathered}
$$

where $\bar{P}_{\mathrm{U}}$ and $\underline{P}_{\mathrm{U}}$ are the upper and lower limits of the system uncertainty set; $\tilde{P}_{\mathrm{C}}^{\max }$ and $\tilde{P}_{\mathrm{C}}^{\mathrm{min}}$ are the upward and downward bounds of the synergistic capability; $\Delta P_{\mathrm{D}}^{\mathrm{rc} 1}, \Delta P_{\mathrm{D}}^{\mathrm{rc} 2}, \Delta P_{\mathrm{W}}^{\mathrm{rc} 1}$, $\Delta P_{\mathrm{W}}^{\mathrm{rc} 2}$ are the respective curtailment of load and wind power when the synergistic capability is maximized. Constraint (11) imposes that the downward bound of the synergistic capability should be less than the lower limit of the system uncertainty set, and constraint (12) imposes that the upward bound of the synergistic capability should be more than the upper limit of the system uncertainty set. Constraints (11) and (12) indicate that the synergistic capability should cover the variation range of loads and wind power.

3) Constraints for conventional units

$$
\begin{aligned}
& \underline{P}_{\mathrm{G} g} \leq \tilde{P}_{\mathrm{G} g}^{\min }=P_{\mathrm{G} g}^{0}+\Delta P_{\mathrm{G} g}^{\mathrm{cdn}}+R_{\mathrm{G} g} \Delta f^{\mathrm{up}} \\
& P_{\mathrm{G} g}^{0}+\Delta P_{\mathrm{G} g}^{\mathrm{cup}}+R_{\mathrm{G} g} \Delta f^{\mathrm{dn}}=\tilde{P}_{\mathrm{G} g}^{\max } \leq \bar{P}_{\mathrm{G} g} \\
& \Delta P_{\mathrm{G} g}^{\mathrm{cdn}}=\beta_{\mathrm{G} g}\left(\underline{P}_{\mathrm{U}}-\beta_{\mathrm{f}} \Delta f^{\mathrm{dn}}+\sum_{j \in N_{\mathrm{D}}} \Delta P_{\mathrm{D} j}^{\mathrm{rc} 1}-\sum_{k \in N_{\mathrm{W}}} \Delta P_{\mathrm{W} k}^{\mathrm{rc} 1}\right)
\end{aligned}
$$

$\Delta P_{\mathrm{G} g}^{\mathrm{cup}}=\beta_{\mathrm{G} g}\left(\bar{P}_{\mathrm{U}}-\beta_{\mathrm{f}} \Delta f^{\mathrm{up}}+\sum_{j \in N_{\mathrm{D}}} \Delta P_{\mathrm{D} j}^{\mathrm{rc} 2}-\sum_{k \in N_{\mathrm{W}}} \Delta P_{\mathrm{W} k}^{\mathrm{rc} 2}\right)$

$-\bar{r}_{\mathrm{G} g}^{\mathrm{dn}} \Delta \tau \leq \Delta P_{\mathrm{G} g}^{\mathrm{cdn}} \leq 0$

$0 \leq \Delta P_{\mathrm{G} g}^{\mathrm{cup}} \leq \bar{r}_{\mathrm{G} g}^{\mathrm{up}} \Delta \tau$

where $\tilde{P}_{\mathrm{G} g}$ is the power output of conventional unit $g ; \bar{P}_{\mathrm{G} g}$ and $\underline{P}_{\mathrm{G} g}$ are the maximum and minimum power output limits of unit $g ; \tilde{P}_{\mathrm{G} g}^{\max }$ and $\tilde{P}_{\mathrm{G} g}^{\min }$ are the maximum and minimum power output bounds of unit $g$ due to the realization of uncertainties; $\beta_{\mathrm{G} g}$ is the participation factor for unit $g, 0 \leq \beta_{\mathrm{G} g}$ and $\sum_{g \in N_{\mathrm{G}}} \beta_{\mathrm{G} g}=1 ; \vec{r}_{\mathrm{G} g}^{\mathrm{up}}$ and $\vec{r}_{\mathrm{G} g}^{\mathrm{dn}}$ are the upward and downward ramp limits for unit $g ; \Delta \tau$ is the response time period, and $\Delta \tau=5 \mathrm{~min}$. Equations (13) and (14) represent the unit capacity constraints, and (15)-(18) represent the unit reserve capability constraints. Equations (15) and (16) indicate that the units share the power imbalance as per their participation factors. Therefore, the expressions of (15) and (16) follow the affine rules [14] mathematically.

4) Constraints for transmission lines

For each transmission line, the flow on it can be expressed as the linear function of nodal injection power [31]. That is, 
$\tilde{P}_{\mathrm{L} l}=\sum_{i \in N_{\mathrm{B}}} \psi_{l, i}\left(\sum_{g \in N_{\mathrm{G}, i}} \tilde{P}_{\mathrm{G} g}+\sum_{k \in N_{\mathrm{W}, i}} \tilde{P}_{\mathrm{W} k}-\sum_{j \in N_{\mathrm{D}, i}} \tilde{P}_{\mathrm{D} j}\right) \quad \forall l \in N_{\mathrm{T}}$

where $\tilde{P}_{\mathrm{L} l}$ is the flow on transmission lines $l ; \psi_{l, i}$ is the injection shift factor (ISF) [31] of power injection at bus $i$ on transmission line $l ; N_{\mathrm{T}}$ is the set of transmission lines.

For the actual closed loop scheme of generation control is based on the concept of participation factors, (19) under the affine policy can be rewritten as

$$
\begin{aligned}
\tilde{P}_{\mathrm{L} l}= & \sum_{i \in N_{\mathrm{B}}} \psi_{l, i}\left(\sum_{g \in N_{\mathrm{G}, i}} P_{\mathrm{G} g}^{0}+\sum_{k \in N_{\mathrm{W}, i}} P_{\mathrm{W} k}^{0}-\sum_{j \in N_{\mathrm{D}, i}} P_{\mathrm{D} j}^{0}\right) \\
& +\sum_{i \in N_{\mathrm{B}}}\left(\psi_{l, i}-\sum_{g(n) \in N_{\mathrm{G}}} \beta_{\mathrm{G} g} \psi_{l, k}\right)\left(\sum_{k \in N_{\mathrm{W}, i}} \Delta \tilde{P}_{\mathrm{W} k}-\sum_{j \in N_{\mathrm{D}, i}} \Delta \tilde{P}_{\mathrm{D} j}\right) \\
& +\sum_{i \in N_{\mathrm{B}}}\left\{\sum_{g \in N_{\mathrm{G}, i}}\left(R_{\mathrm{G} g}-\beta_{\mathrm{G} g} \beta_{\mathrm{f}}\right)-\sum_{j \in N_{\mathrm{D}, i}} D_{\mathrm{D} j}\right\} \psi_{l, i} \Delta \tilde{f} \\
= & P_{\mathrm{L} l}^{0}+\sum_{i \in N_{\mathrm{B}}} \varphi_{l, i} \Delta \tilde{P}_{\mathrm{I} i}+\varphi_{l, \mathrm{f}} \Delta \tilde{f}
\end{aligned}
$$

$$
\left\{\begin{array}{l}
P_{\mathrm{L} l}^{0} \triangleq \sum_{i \in N_{\mathrm{B}}} \psi_{l, i}\left(\sum_{g \in N_{\mathrm{G}, i}} P_{\mathrm{G} g}^{0}+\sum_{k \in N_{\mathrm{W}, i}} P_{\mathrm{W} k}^{0}-\sum_{j \in N_{\mathrm{D}, i}} P_{\mathrm{D} j}^{0}\right) \\
\Delta \tilde{P}_{\mathrm{I} i} \triangleq \sum_{k \in N_{\mathrm{W}, i}} \Delta \tilde{P}_{\mathrm{W} k}-\sum_{j \in N_{\mathrm{D}, i}} \Delta \tilde{P}_{\mathrm{D} j} \\
\varphi_{l, i} \triangleq \psi_{1, i}-\sum_{g(n) \in N_{\mathrm{G}}} \beta_{\mathrm{G} g} \psi_{l, n} \\
\varphi_{l, \mathrm{f}} \triangleq \sum_{i \in N_{\mathrm{B}}}\left\{\sum_{g \in N_{\mathrm{G}, i}}\left(R_{\mathrm{G} g}-\beta_{\mathrm{G} g} \beta_{\mathrm{f}}\right)-\sum_{j \in N_{\mathrm{D}, i}} D_{\mathrm{D} j}\right\} \psi_{l, i}
\end{array}\right.
$$

where $g(n)$ is the $g^{\text {th }}$ unit located at bus $n$; " $\triangleq$ " is the definition symbol; $\Delta \tilde{P}_{\mathrm{I} i}$ is the injection uncertainty of bus $i ; \varphi_{l, i}$ is the power transfer distribution factor (PTDF) [31] of power flow on transmission line $l$ with the variation of the $i$ th bus's injection power; $\varphi_{l, \mathrm{f}}$ is the sensitivity factor of power flow on transmission line $l$ with the variation of the system frequency.

Therefore, the transmission constraints could be written as

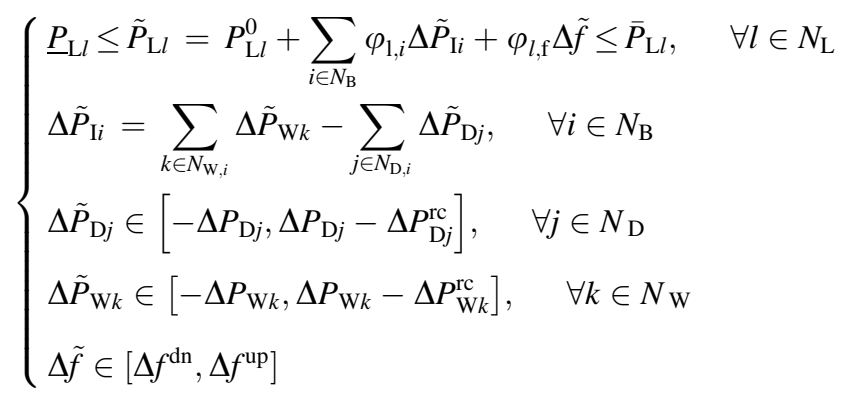

where $\bar{P}_{\mathrm{L} l}$ and $\underline{P}_{\mathrm{L} l}$ are the maximum and minimum power transmission limits of transmission line $l$.

Equation (22) follows the affine rules, and can be regarded as the interval inequality constraints with the variable ranges of the uncertain parameters from the view of uncertain linear programming [14]. Additionally, it implies that the worst realization of uncertainty sets should be satisfied. The maximum and minimum power of a transmission line can be achieved only when the nodal injection uncertainty reaches its upper or lower bounds [10]. If one nodal injection uncertainty of bus $i$ is $\Delta \tilde{P}_{i} \in\left[\underline{\underline{P}} \underline{ }_{i}, \Delta \bar{P}_{i}\right]$, the maximum contribution of it to transmission constraints for transmission line $l$ will be on the bound of the uncertainty set, depending on the sign of $\varphi_{l, i}$. That is,

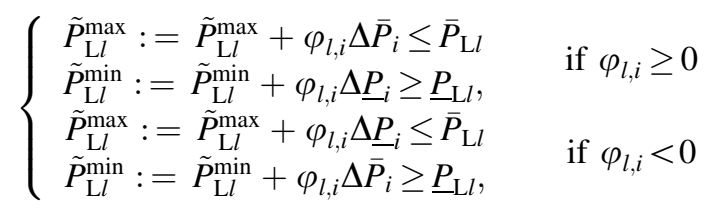

where $\tilde{P}_{\mathrm{L} l}^{\max }$ and $\tilde{P}_{\mathrm{L} l}^{\min }$ are the maximum and minimum flow on transmission line $l$ due to the realization of uncertainties; " $:="$ is the assigning operator. For clarity, if two nodal injection uncertainties are considered, then (23) is expanded below:

$$
\left\{\begin{aligned}
\tilde{P}_{\mathrm{L} l}^{\max }:=\tilde{P}_{\mathrm{L} l}^{\max }+\varphi_{l, i} \Delta \bar{P}_{i}+\varphi_{l, n} \Delta \bar{P}_{n} \leq \bar{P}_{\mathrm{L} l} & \\
\tilde{P}_{\mathrm{L} l}^{\min }:=\tilde{P}_{\mathrm{L} l}^{\min }+\varphi_{l, i} \Delta \underline{P}_{i}+\varphi_{l, n} \Delta \bar{P}_{n} \geq \underline{P}_{\mathrm{L} l}, & \text { if } \varphi_{l, i} \geq 0, \varphi_{l, n} \geq 0 \\
\tilde{P}_{\mathrm{L} l}^{\max }:=\tilde{P}_{\mathrm{Ll}}^{\max }+\varphi_{l, i} \Delta \bar{P}_{i}+\varphi_{l, n} \Delta \underline{P}_{n} \leq \bar{P}_{\mathrm{L} l} & \\
\tilde{P}_{\mathrm{L} l}^{\min }:=\tilde{P}_{\mathrm{Ll}}^{\min }+\varphi_{l, i} \Delta \underline{P}_{i}+\varphi_{l, n} \Delta \bar{P}_{n} \geq \underline{P}_{\mathrm{L} l}, & \text { if } \varphi_{l, i} \geq 0, \varphi_{l, n}<0 \\
\tilde{P}_{\mathrm{L} l}^{\max }:=\tilde{P}_{\mathrm{L} l}^{\max }+\varphi_{l, i} \underline{P}_{i}+\varphi_{l, n} \Delta \bar{P}_{n} \leq \bar{P}_{\mathrm{L} l}, & \text { if } \varphi_{l, i}<0, \varphi_{l, n} \geq 0 \\
\tilde{P}_{\mathrm{L} l}^{\min }:=\tilde{P}_{\mathrm{L} l}^{\min }+\varphi_{l, i} \Delta \bar{P}_{i}+\varphi_{l, n} \Delta \underline{P}_{n} \geq \underline{P}_{\mathrm{L} l} & \\
\tilde{P}_{\mathrm{L} l}^{\max }:=\tilde{P}_{\mathrm{Ll}}^{\max }+\varphi_{l, i} \Delta \underline{P}_{i}+\varphi_{l, n} \Delta \underline{P}_{n} \leq \bar{P}_{\mathrm{L} l} & \\
\tilde{P}_{\mathrm{L} l}^{\min }:=\tilde{P}_{\mathrm{L} l}^{\min }+\varphi_{l, i} \Delta \bar{P}_{i}+\varphi_{l, n} \Delta \bar{P}_{n} \geq \underline{P}_{\mathrm{L} l}, & \text { if } \varphi_{l, i}<0, \varphi_{l, n}<0
\end{aligned}\right.
$$

This process will be repeated for all the nodal injection uncertainties. However, the signs of the variables $\varphi_{l, i}$ and $\varphi_{l, \mathrm{f}}$ are unknown and they depend on the balance mechanism for injection uncertainties, to be exact, the participation factors of conventional units shown in (21). Therefore, all possible combinations of signs of $\varphi_{l, i}$ and $\varphi_{l, n}$ should be considered to immunize all possible scenario realizations under interval uncertainties [10]. Thus the number of transmission constraints will be doubled for one more nodal injection uncertainty. Assume that the number of transmission lines is $n l$, and the number of nodal injection uncertainties is $n$, then there will be $2^{n+1} n l$ combinatorial transmission constraints to be regarded in principle. 
5) Other constraints

$$
\begin{aligned}
& \Delta f_{-} \leq \Delta f^{\mathrm{dn}} \leq 0 \\
& 0 \leq \Delta f^{\mathrm{up}} \leq \Delta \bar{f} \\
& \left\{\begin{array}{l}
\Delta P_{\mathrm{D} j}^{\mathrm{rc}} \leq \Delta \bar{P}_{\mathrm{D} j}^{\mathrm{rc}} \\
0 \leq \Delta P_{\mathrm{D} j}^{\mathrm{rc} 1} \leq \Delta P_{\mathrm{D} j}^{\mathrm{rc}}, \quad \forall j \in N_{\mathrm{D}} \\
0 \leq \Delta P_{\mathrm{D} j}^{\mathrm{rc} 2} \leq \Delta P_{\mathrm{D} j}^{\mathrm{rc}}
\end{array}\right. \\
& \left\{\begin{array}{l}
\Delta P_{\mathrm{W} k}^{\mathrm{rc}} \leq \Delta \bar{P}_{\mathrm{W} k}^{\mathrm{rc}} \\
0 \leq \Delta P_{\mathrm{W} k}^{\mathrm{rc}} \leq \Delta P_{\mathrm{W} k}^{\mathrm{rc}}, \quad \forall k \in N_{\mathrm{W}} \\
0 \leq \Delta P_{\mathrm{W} k}^{\mathrm{rc} 2} \leq \Delta P_{\mathrm{W} k}^{\mathrm{rc}}
\end{array}\right.
\end{aligned}
$$

where $\Delta f$ and $\Delta \bar{f}$ are the allowable lower and upper limits of system frequency deviation, which imply the range of the automatic synergistic capability in power system operation; $\Delta \bar{P}_{\mathrm{D}}^{r c}$ and $\Delta \bar{P}_{\mathrm{W}}^{r c}$ are the allowable upper limits for curtailment of load and wind power, respectively.

\section{Solution methodology for FARED}

This section gives the solution method to improve the computational tractability of the robust optimization formulation for FARED problem.

\subsection{Relationship between FARED and traditional economic dispatch}

The model of FARED is compatible with the traditional economic dispatch model. If the allowable frequency upward and downward deviations are 0 , and the injection uncertainties are tackled by the secondary reserve of units, the proposed model is simplified to be the robust optimization counterpart of traditional economic dispatch with the non-negative variables $P_{\mathrm{G}}^{0}$ and $\beta_{\mathrm{G}}$ as the decision variables. Then the simplified model with given uncertainty sets can be transformed into a quadratic programming (QP) and solved by the state-of-theart QP solution methods [32]. However, due to the allowable frequency deviation and possible load or renewable generation curtailments, the formulation for the FARED problem is not a standard robust optimization problem [15] because of the variable uncertainty sets, but it can be converted into a nonlinear programming (NLP) for the bilinear terms in unit reserve capability constraints constraints and transmission constraints, then it can be solved by available bilinear programming (BLP) solution methodologies [33].

\subsection{Extraction of dominant constraints}

With the increase of the number of injection uncertainties, the number of transmission constraints exponentially increase. This will cause an NP-hard problem for the large-scale power systems. However, only two constraints in (23) and (24) can be dominant constraints that are the candidates of tight transmission constraints, depending on the signs of the PTDFs shown in (21). Equations (23) and (24) indicate that the system should accommodate the worst scenario realization of branch flow under injection uncertainties for safe operation. By duality theory, expressions (23) can be transformed into the following set of complementarity constraints [34].

$$
\left\{\begin{array}{l}
\tilde{P}_{\mathrm{L} l}^{\max }:=\tilde{P}_{\mathrm{L} l}^{\max }+\varphi_{l, i} \Delta P_{i} \leq \bar{P}_{\mathrm{L} l} \\
\tilde{P}_{\mathrm{L} l}^{\min }:=\tilde{P}_{\mathrm{L} l}^{\min }+\varphi_{l, i}\left(\Delta \underline{P}_{i}+\Delta \bar{P}_{i}-\Delta P_{i}\right) \geq \underline{P}_{\mathrm{L} l} \\
\varphi_{l, i}\left(\Delta P_{i}-\Delta \bar{P}_{i}\right) \geq 0 \\
\varphi_{l, i}\left(\Delta P_{i}-\Delta \underline{P}_{i}\right) \geq 0 \\
\Delta \underline{P}_{i} \leq \Delta P_{i} \leq \Delta \bar{P}_{i}
\end{array}\right.
$$

Similarly, expressions (24) can be transformed as:

$$
\left\{\begin{array}{l}
\tilde{P}_{\mathrm{L} l}^{\max }:=\tilde{P}_{\mathrm{L} l}^{\max }+\varphi_{l, i} \Delta P_{i}+\varphi_{l, k} \Delta P_{k} \leq \bar{P}_{\mathrm{L} l} \\
\tilde{P}_{\mathrm{L} l}^{\min }:=\tilde{P}_{\mathrm{L} l}^{\min }+\varphi_{l, i}\left(\Delta \underline{P}_{i}+\Delta \bar{P}_{i}-\Delta P_{i}\right)+\varphi_{l, k}\left(\Delta \underline{P}_{k}+\Delta \bar{P}_{k}-\Delta P_{k}\right) \geq \underline{P}_{\mathrm{L} l} \\
\varphi_{l, i}\left(\Delta P_{i}-\Delta \bar{P}_{i}\right) \geq 0 \\
\varphi_{l, i}\left(\Delta P_{i}-\Delta \underline{P}_{i}\right) \geq 0 \\
\Delta \underline{P}_{i} \leq \Delta P_{i} \leq \Delta \bar{P}_{i} \\
\varphi_{l, k}\left(\Delta P_{k}-\Delta \bar{P}_{k}\right) \geq 0 \\
\varphi_{l, k}\left(\Delta P_{k}-\Delta \underline{P}_{k}\right) \geq 0 \\
\Delta \underline{P}_{k} \leq \Delta P_{k} \leq \Delta \bar{P}_{k}
\end{array}\right.
$$

This process will be repeated for all the nodal injection uncertainties. Thus with the extraction of dominant constraints, the number of transmission constraints will be reduced to $2 n l(n+1)+2 n$. It is worth mentioning that with the introduction of complementarity constraints, the formulation for FARED still belongs to the bilinear programming.

\subsection{Curtailment of constraints}

One intuitive means for reducing constraints is to determine the set of transmission lines considered for transmission constraints by engineering experience or based on a simplified power scheduling in which transmission constraints are not considered.

Additionally, the model can be further simplified by reducing the number of transmission constraints. Since the ISFs are parameters and the participation factors of conventional units belong to $[0,1]$, the signs of some PTDFs in (21) can be determined before optimization. The IEEE 9-bus system is shown in Fig. 1. $\varphi_{5-6,5}$ is always positive, and $\varphi_{8-9,9}$ always negative, that is to say, the increase of injection power for $\mathrm{Bus}_{5}$ will always increase the flow on transmission Line 5-6, whereas the increase of injection power for Bus 9 will always decrease the flow on transmission Line 8-9. If the signs of some PTDFs can be determined before optimization, the model can be further simplified, and the computation burden is well reduced. 


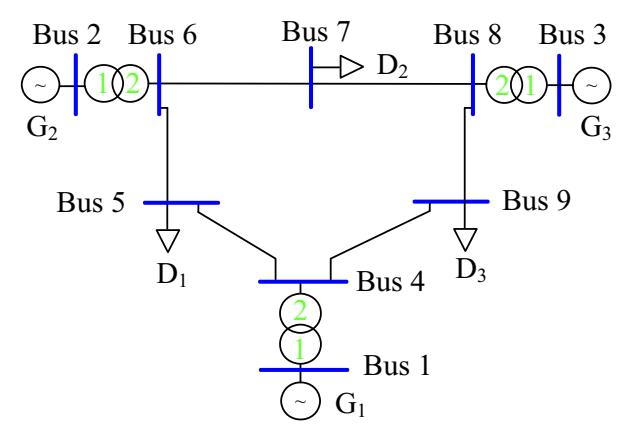

Fig. 1 IEEE 9-bus system

\subsection{Solution procedure}

The solution procedure is as follows.

1) Compute the ISFs based on the result of topological analysis.

2) Determine the set of transmission lines considered for transmission constraints based on the optimal results of economic dispatch without regard to transmission constraints, and reduce the transmission constraints on the basis of the signs of PTDFs.

3) Extract the dominant constraints for network security.

4) Solve the NLP problem using available solution methodologies.

\section{Case studies}

The numerical studies are conducted on the modified IEEE 9-bus and 118-bus system, followed by comparative analysis of computational efficiency. The simulations are performed on the general algebraic modeling system (GAMS) [35] platform, and the BLP problem is solved by CONOPT 3 solver on a PC with a $3.10 \mathrm{GHz}$ processor and $8 \mathrm{G}$ bytes of RAM. For all cases studied, it is assumed that for each unit $g, g \in N_{\mathrm{G}}, R_{\mathrm{G} g}=4 \%$, i.e., a $100 \%$ change in unit output requires a $4 \%$ change in frequency, and for each load $j, j \in N_{\mathrm{D}}, D_{\mathrm{D} j}=2.89$ [36], i.e., load changed by $2.89 \%$ for a $1 \%$ change in frequency. $\pm 2.5 \%$ load uncertainty is assumed. The nominal frequency is $50 \mathrm{~Hz}$.

In order to illustrate the effectiveness of the proposed method, we take the following four scheduling schemes for comparative analysis: (1) classical economic dispatch (CED) without regard to transmission constraints. The system frequency is fixed to the nominal value; (2) synergetic economic dispatch (SED) [19] without regard to transmission constraints. The allowable frequency variation range is $\pm 0.04 \mathrm{~Hz}$; (3) robust economic dispatch (RED), viz., the proposed FARED method with the system frequency fixed to the nominal value; (4) the proposed
FARED method with the allowable frequency variation range of $\pm 0.04 \mathrm{~Hz}$.

\subsection{Case study on modified IEEE 9-bus system}

The modified IEEE 9-bus system with wind power integration (IEEE-9W) is shown in Fig. 2. The parameters of transmission lines, characteristics of conventional units and data for injection uncertainties are shown in Table A1, A2, A3 in Appendix A.

1) IEEE 9-bus system

The results of power scheduling for the IEEE 9-bus system are listed in Table 1.

For CED and RED, the uncertainties are tackled by the secondary reserve, which corresponds to the activation of the controllable synergistic capability. From Table 1, it can be found that $\pm 7.875 \mathrm{MW}$ spinning reserve is assigned. However, for SED and FARED, the variation of load power is covered by the automatic synergistic capability. When the deviation of the actual load power from the predicted value is $7.875 \mathrm{MW}$, the power balance is achieved on the condition of the allowable frequency deviation of $0.035 \mathrm{~Hz}$, and $\pm 7.175 \mathrm{MW}$ spinning reserve is assigned due to accounting for the participation of loads in response to the frequency deviation. Therefore, the spinning reserve requirement can be well reduced when considering the frequency related automatic synergistic capability. The comparison of CED with RED, and SED with FARED shows that the transmission line 8-9 is overloaded for both CED and SED with the maximum load ratio being 1.085 and 1.047 , respectively. When transmission constraints are considered, the adjustment of base-point generation of units is performed, and the costs of generation increase accordingly. The comparison of RED and FARED shows that the latter has the advantage of lower generation cost and less spinning reserve requirement.

2) Modified IEEE 9-bus system with wind power integration

The results of power scheduling for the modified IEEE 9-bus system with wind power integration are shown in Table 2.

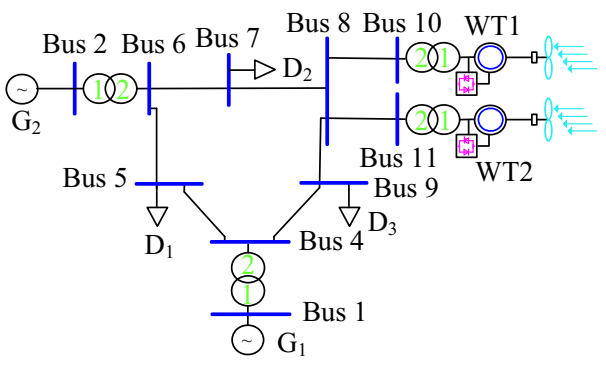

Fig. 2 Modified IEEE 9-bus system with wind power integration 
Table 1 Results of power scheduling for IEEE 9-bus system

\begin{tabular}{|c|c|c|c|c|c|c|c|c|c|c|c|c|c|c|}
\hline \multirow[t]{2}{*}{ Method } & \multicolumn{3}{|c|}{$\begin{array}{l}\text { Base-point generation } \\
\text { (MW) }\end{array}$} & \multicolumn{3}{|c|}{$\begin{array}{l}\text { Upward } \\
\text { secondary } \\
\text { regulation } \\
\text { reserve (MW) }\end{array}$} & \multicolumn{3}{|c|}{$\begin{array}{l}\text { Downward } \\
\text { secondary } \\
\text { regulation } \\
\text { reserve (MW) }\end{array}$} & \multirow[t]{2}{*}{$\Delta f^{\text {up }}$} & \multirow[t]{2}{*}{$\Delta f^{\mathrm{dn}}$} & \multirow[t]{2}{*}{$\begin{array}{l}\text { Unit primary } \\
\text { frequency power } \\
\text { component } \\
\text { (MW) }\end{array}$} & \multirow[t]{2}{*}{$\begin{array}{l}\text { Maximum } \\
\text { branch load } \\
\text { ratio }\end{array}$} & \multirow[t]{2}{*}{$\begin{array}{l}\text { Cost } \\
\text { (\$/hour) }\end{array}$} \\
\hline & $\mathrm{G}_{1}$ & $\mathrm{G}_{2}$ & $\mathrm{G}_{3}$ & $\mathrm{G}_{1}$ & $\mathrm{G}_{2}$ & $\mathrm{G}_{3}$ & $\mathrm{G}_{1}$ & $\mathrm{G}_{2}$ & $\mathrm{G}_{3}$ & & & & & \\
\hline CED & 86.56 & 94.06 & 134.38 & 0 & 0 & 7.875 & 0 & 0 & 7.875 & 0 & 0 & 0 & 1.085 & 5636.55 \\
\hline SED & 86.56 & 94.06 & 134.38 & 0 & 0 & 0 & 0 & 0 & 0 & 0.035 & -0.035 & 7.175 & 1.047 & 5216.03 \\
\hline RED & 96.05 & 93.65 & 125.30 & 0 & 0 & 7.875 & 0 & 0 & 7.875 & 0 & 0 & 0 & 1 & 5653.49 \\
\hline FARED & 93.86 & 93.74 & 127.40 & 0 & 0 & 0 & 0 & 0 & 0 & 0.035 & -0.035 & 7.175 & 1 & 5226.04 \\
\hline
\end{tabular}

As shown in Table 2, for CED and RED all the conventional units reach the maximum reserve capacity, namely $\pm 26 \mathrm{MW}$, but the variation range of loads and wind power is still unable to be covered. Curtailments of loads and wind power are inevitable, which corresponds to the activation of the re-controllable synergistic capability. When the variation reaches $-27.875 \mathrm{MW}, 1.875 \mathrm{MW}$ of loads needs to be curtailed. Similarly, $1.875 \mathrm{MW}$ of wind power needs to be abandoned when the variation is 27.875 MW. Whereas for SED and FARED, the automatic synergistic capability with the range of frequency deviation $\pm 0.04 \mathrm{~Hz}$ is fully utilized, no load or wind power curtailment is required, and correspondingly $\pm 27.15 \mathrm{MW}$ spinning reserve is assigned due to accounting for the participation of loads in response to the frequency deviation. The comparison between CED with RED, and SED with FARED shows that the transmission line 8-9 is overloaded for both CED and SED with the maximum load ratio being 1.222 and 1.213 , respectively. When transmission constraints are considered, base-point generation of units is adjusted, and the production cost increases accordingly. The comparison of RED and FARED shows that the latter takes the frequency deviation as the decision variable, so the automatic synergistic capability can be well considered. Thus the solution space of power scheduling is expanded, and the amount of load or wind power curtailment is reduced effectively.

\subsection{Case study on modified IEEE 118-bus system with wind power integration}

Another bigger case study is conducted on the modified IEEE 118-bus system concerning wind power penetration (IEEE-118W). The data for wind farms is shown in Table B1 in Appendix B. The test system is comprised of 118 buses, 19 generators, 99 loads and 186 transmission lines [37]. The scheduling results are listed in Table 3.

Table 3 shows that the overloaded transmission lines appear for both classical economic dispatch and the synergetic economic dispatch. For both CED and SED, the set of overloaded transmission lines is $\{112,115,140\}$, with the maximum load ratio being 1.593 and 1.368 , respectively. However, the robust economic dispatch scheme and the frequency aware robust economic dispatch scheme can ensure transmission security effectively, and the latter has the advantage of lower cost, and less spinning reserve requirement for considering the automatic synergistic capability.

\subsection{Comparative analysis of computational efficiency}

In order to illustrate the computational efficiency, the IEEE 14-bus, 30-bus and 118-bus systems [37] are considered as the examples besides the above-mentioned

Table 2 Results of power scheduling for modified IEEE 9-bus system with wind power integration

\begin{tabular}{|c|c|c|c|c|c|c|c|c|c|c|c|c|c|c|c|c|}
\hline \multirow[t]{2}{*}{ Method } & \multicolumn{2}{|c|}{$\begin{array}{l}\text { Base-point } \\
\text { generation } \\
\text { (MW) }\end{array}$} & \multicolumn{2}{|c|}{$\begin{array}{l}\text { Upward } \\
\text { secondary } \\
\text { regulation } \\
\text { reserve } \\
\text { (MW) }\end{array}$} & \multicolumn{2}{|c|}{$\begin{array}{l}\text { Downward } \\
\text { secondary } \\
\text { regulation } \\
\text { reserve } \\
\text { (MW) }\end{array}$} & \multicolumn{2}{|c|}{$\begin{array}{l}\text { Wind power } \\
\text { curtailment } \\
(\mathrm{MW})\end{array}$} & \multicolumn{3}{|c|}{$\begin{array}{l}\text { Load } \\
\text { curtailment } \\
\text { (MW) }\end{array}$} & \multirow[t]{2}{*}{$\Delta f^{\text {up }}$} & \multirow[t]{2}{*}{$\Delta f^{\mathrm{dn}}$} & \multirow[t]{2}{*}{$\begin{array}{l}\text { Unit } \\
\text { primary } \\
\text { frequency } \\
\text { power } \\
\text { component } \\
\text { (MW) }\end{array}$} & \multirow[t]{2}{*}{$\begin{array}{l}\text { Maximum } \\
\text { branch } \\
\text { load ratio }\end{array}$} & \multirow[t]{2}{*}{$\begin{array}{l}\text { Cost } \\
\text { (\$/hour) }\end{array}$} \\
\hline & $\mathrm{G}_{1}$ & $\mathrm{G}_{2}$ & $\mathrm{G}_{1}$ & $\mathrm{G}_{2}$ & $\mathrm{G}_{1}$ & $\mathrm{G}_{2}$ & WT1 & WT2 & $\mathrm{D}_{1}$ & $\mathrm{D}_{2}$ & $\mathrm{D}_{3}$ & & & & & \\
\hline CED & 83.60 & 91.40 & 12.5 & 13.5 & 12.5 & 13.5 & 1.875 & 0 & 0 & 0 & 1.875 & 0 & 0 & 0 & 1.222 & 4789.07 \\
\hline SED & 83.60 & 91.40 & 12.5 & 9.45 & 12.5 & 9.45 & 0 & 0 & 0 & 0 & 0 & 0.04 & -0.04 & 5.2 & 1.213 & 4242.84 \\
\hline RED & 99.52 & 75.48 & 12.5 & 13.5 & 12.5 & 13.5 & 1.875 & 0 & 0 & 0 & 1.875 & 0 & 0 & 0 & 1 & 4847.95 \\
\hline FARED & 101.29 & 73.71 & 12.5 & 9.45 & 12.5 & 9.45 & 0 & 0 & 0 & 0 & 0 & 0.04 & -0.04 & 5.2 & 1 & 4315.58 \\
\hline
\end{tabular}


Table 3 Results of power scheduling for modified IEEE 118-bus system with wind power integration

\begin{tabular}{|c|c|c|c|c|c|c|c|c|}
\hline Method & $\begin{array}{l}\text { Upward secondary } \\
\text { regulation reserve } \\
\text { (MW) }\end{array}$ & $\begin{array}{l}\text { Downward } \\
\text { secondary } \\
\text { regulation reserve } \\
(\mathrm{MW})\end{array}$ & $\begin{array}{l}\text { Frequency } \\
\text { deviation } \\
(\mathrm{Hz})\end{array}$ & $\begin{array}{l}\text { Unit primary } \\
\text { frequency power } \\
\text { component (MW) }\end{array}$ & $\begin{array}{l}\text { Wind power } \\
\text { curtailment } \\
(\mathrm{MW})\end{array}$ & $\begin{array}{l}\text { Load } \\
\text { curtailment } \\
\text { (MW) }\end{array}$ & $\begin{array}{l}\text { Maximum } \\
\text { branch load } \\
\text { ratio }\end{array}$ & $\begin{array}{l}\text { Cost } \\
\text { (\$/hour) }\end{array}$ \\
\hline CED & 172.975 & 172.975 & 0 & 0 & 0 & 0 & 1.593 & 3177.76 \\
\hline SED & 45.343 & 45.343 & \pm 0.04 & 117.184 & 0 & 0 & 1.368 & 2727.37 \\
\hline RED & 172.975 & 172.975 & 0 & 0 & 0 & 0 & 1 & 3255.15 \\
\hline FARED & 45.343 & 45.343 & \pm 0.04 & 117.184 & 0 & 0 & 1 & 2746.11 \\
\hline
\end{tabular}

Table 4 Comparison of solution efficiency for FARED

\begin{tabular}{|c|c|c|c|c|c|c|}
\hline \multirow[t]{2}{*}{ Case } & \multicolumn{2}{|c|}{ Cost (\$/hour) } & \multicolumn{2}{|c|}{ Computing time (s) } & \multicolumn{2}{|c|}{ Memory usage (Mb) } \\
\hline & NoEDC & EDC & NoEDC & EDC & NoEDC & EDC \\
\hline IEEE-9 & 5226.04 & 5226.04 & 0.109 & 0.015 & 1.22 & 0.98 \\
\hline IEEE-9W & 4315.58 & 4315.58 & 0.125 & 0.093 & 52.24 & 1.01 \\
\hline IEEE-14 & 7645.21 & 7645.21 & 4.415 & 0.094 & 503.09 & 2.91 \\
\hline IEEE-30 & & 565.21 & & 0.202 & & 5.32 \\
\hline IEEE-118 & & 2914.54 & & 0.421 & & 40.52 \\
\hline IEEE-118W & & 2746.11 & & 16.19 & & 117.31 \\
\hline
\end{tabular}

cases. The comparative analysis of solution efficiency is conducted for the FARED formulation without regard to the extraction of dominant constraints (NoEDC) and the one with the consideration of the extraction of dominant constraints (EDC), and the results are listed in Table 4.

As shown in Table 4, with the increase of the power system size, both the computing time and memory usage increase nonlinearly for the two schemes, and the increase for NoEDC is far more than that for EDC. With the increasing scale of power systems, EDC has better performance. For the IEEE-30, IEEE-118 and IEEE-118W cases, solution by NoEDC is beyond the computing capability of the PC used for simulation, whereas it is computational tractable by EDC. Therefore, the computational efficiency is well improved when the extraction of dominant constraints is considered.

\section{Conclusion and discussions}

In this paper, a frequency aware robust economic dispatch is proposed to deal with the increasing injection uncertainties. In the proposed method, the frequency regulation effect and the unit reserve response mechanism are well considered to exploit the synergistic capability to accommodate the uncertain loads and renewable generations on the decision-making level. The FARED problem is formulated as a robust optimization problem with variable uncertainty sets under the affine policy. Duality theory is used to form complementarity constraints to improve the computational tractability. The test results of FARED show that the proposed method is of benefit to the coordination of dispatch and generation control, as well as the accommodation of large scale renewable power penetration with the advantage of lower cost and less spinning reserve requirement. The computational efficiency of the proposed algorithm is well improved with the extraction of dominant constraints and curtailment of constraints.

This work will be extended to a dynamic FARED with temporal coupling constraints to exploit the synergistic capability of accommodating the injection uncertainties as well as alleviating the unit ramp rate constraints, and dynamic FARED with optimal conventional unit on/off statuses decision-making will be considered. Moreover, the economic participation mechanism of individual resources in FARED under the deregulated electric power markets will be analyzed. Last but not least, the high-performance solution methodologies for the FARED problem are worthy of further study.

Acknowledgements This work was supported by the National Basic Research Program (973 Program) (No. 2013CB228205), and the National Natural Science Foundation of China (No. 51177091, No. 51477091).

Open Access This article is distributed under the terms of the Creative Commons Attribution 4.0 International License (http:// creativecommons.org/licenses/by/4.0/), which permits unrestricted use, distribution, and reproduction in any medium, provided you give appropriate credit to the original author(s) and the source, provide a link to the Creative Commons license, and indicate if changes were made. 


\section{Appendix A}

See Tables A1, A2 and A3.

Table A1 Branch parameters for modified IEEE 9-bus system

\begin{tabular}{lll}
\hline Branch & Reactance & Capacity (MVA) \\
\hline $1-4$ & 0.0576 & 130 \\
$2-6$ & 0.0586 & 140 \\
$3-8$ & 0.0625 & 160 \\
$5-6$ & 0.17 & 100 \\
$4-5$ & 0.092 & 100 \\
$6-7$ & 0.1008 & 100 \\
$7-8$ & 0.072 & 100 \\
$8-9$ & 0.161 & 70 \\
$9-4$ & 0.085 & 100 \\
$10-8$ & 0.092 & 100 \\
$11-8$ & 0.092 & 100 \\
\hline
\end{tabular}

Table A2 Unit parameters for modified IEEE 9-bus system

\begin{tabular}{llllllll}
\hline Bus & $\begin{array}{l}\bar{P}_{\mathrm{G}} \\
(\mathrm{MW})\end{array}$ & $\begin{array}{l}\underline{P}_{\mathrm{G}} \\
(\mathrm{MW})\end{array}$ & $R_{\text {ramp }}(\mathrm{MW} / \mathrm{min})$ & \multicolumn{3}{l}{ Cost $(\$ /$ hour $)$} & \\
\hline 1 & 125 & 50 & 2.5 & $a_{\mathrm{G}}$ & $b_{\mathrm{G}}$ & $c_{\mathrm{G}}$ \\
\hline 2 & 135 & 54 & 2.7 & 0.11 & 5 & 150 \\
3 & 150 & 60 & 3 & 0.1225 & 1 & 335 \\
\hline
\end{tabular}

Table A3 Injection power for modified IEEE 9-bus system

\begin{tabular}{lcc}
\hline Bus & $\underline{P}(\mathrm{MW})$ & $\bar{P}(\mathrm{MW})$ \\
\hline 5 & -92.25 & -87.75 \\
7 & -102.5 & -97.5 \\
9 & -128.125 & -121.875 \\
10 & 60 & 80 \\
11 & 60 & 80 \\
\hline
\end{tabular}

\section{Appendix B}

See Table B1.

Table B1 Wind power for modified IEEE 118-bus system

\begin{tabular}{lcc}
\hline Bus & $\underline{P}_{\mathrm{W}}(\mathrm{MW})$ & $\bar{P}_{\mathrm{W}}(\mathrm{MW})$ \\
\hline 1 & 60 & 80 \\
19 & 60 & 80 \\
56 & 40 & 60 \\
73 & 90 & 110 \\
112 & 110 & 130 \\
116 & 110 & 130 \\
\hline
\end{tabular}

\section{References}

[1] Wood AJ, Wollenberg BF (1996) Power generation, operation and control, 2nd edn. Wiley, New York, pp 29-123, 209-230

[2] Dommel HW, Tinney WF (1968) Optimal power flow solutions. IEEE Trans Power Appar Syst 87(10):1866-1876

[3] Mukai H, Singh J, Spare J et al (1981) A reevaluation of the normal operating state control of the power system using computer control and system theory-Part II: dispatch targeting. IEEE Trans Power Appar Syst 100(1):309-317

[4] Kambale P, Mukai H, Spare J et al (1983) A reevaluation of the normal operating state control of the power system using computer control and system theory-Part III: tracking the dispatch targets with unit control. IEEE Trans Power Appara Syst 102(6): 1903-1912

[5] Smith JC, Milligan MR, DeMeo EA et al (2007) Utility wind integration and operating impact state of the art. IEEE Trans Power Syst 22(3):900-908

[6] Zhang H, Li P (2011) Chance constrained programming for optimal power flow under uncertainty. IEEE Trans Power Syst 26(4):2417-2424

[7] Lee YY, Baldick R (2013) A frequency-constrained stochastic economic dispatch model. IEEE Trans Power Syst 28(3): 2301-2312

[8] Yan HZ, Luh PB (1997) A fuzzy optimization-based method for integrated power system scheduling and inter-utility power transaction with uncertainties. IEEE Trans Power Syst 12(2): 756-763

[9] Attaviriyanupap P, Kita H, Tanaka E et al (2004) A fuzzyoptimization approach to dynamic economic dispatch considering uncertainties. IEEE Trans Power Syst 19(3):1299-1307

[10] Saric AT, Stankovic AM (2006) An application of interval analysis and optimization to electric energy markets. IEEE Trans Power Syst 21(2):515-523

[11] Ding T, Bo R, Gu W et al (2014) Absolute value constraint based method for interval optimization to SCED model. IEEE Trans Power Syst 29(2):980-981

[12] Han XS, Han L, Gooi HB et al (2012) Ultra-short-term multinode load forecasting - a composite approach. IET Gener Transm Distrib 6(5):436-444

[13] Wan C, Xu Z, Pinson P et al (2014) Optimal prediction intervals of wind power generation. IEEE Trans Power Syst 29(3): $1166-1174$

[14] Ben-Tal A, Goryashko A, Guslitzer E et al (2004) Adjustable robust solutions of uncertain linear programs. Math Program 99(2):351-376

[15] Bertsimas D, Brown DB, Caramanis C (2011) Theory and applications of robust optimization. SIAM Rev 53(3):464-501

[16] Rouholamini M, Rashidinejad M, Abdollahi A et al (2012) Frequency reserve within unit commitment considering spinning reserve uncertainty. Int J Energy Eng 2(4):177-183

[17] Bertsimas D, Litvinov E, Sun XA et al (2013) Adaptive robust optimization for the security constrained unit commitment problem. IEEE Trans Power Syst 28(1):52-63

[18] Wang SB, Han XS, Yang M (2013) Interval economic dispatch of power system considering unit reserve responsiveness. Proc CSEE 33(7):99-108 (in Chinese)

[19] Liu GJ, Han XS, Yang M (2014) Synergetic economic dispatch in power system operation. Proc CSEE 34(16):2668-2675 (in Chinese)

[20] Jabr RA (2013) Adjustable robust OPF with renewable energy sources. IEEE Trans Power Syst 28(4):4742-4751

[21] Yang M, Cheng FL, Han XS (2015) Real-time dispatch based on effective steady-state security regions of power system. Proc CSEE 35(6):1353-1362 (in Chinese) 
[22] Chavez H, Baldick R, Sharma S (2014) Governor rate-constrained OPF for primary frequency control adequacy. IEEE Trans Power Syst 29(3):1473-1480

[23] Zhang GY, McCalley J (2014) Optimal power flow with primary and secondary frequency constraint. In: Proceedings of the North American power symposium (NAPS'14), Pullman, 7-9 Sept 2014, 6 pp

[24] Ahmadi H, Ghasemi H (2014) Security-constrained unit commitment with linearized system frequency limit constraints. IEEE Trans Power Syst 29(4):1536-1545

[25] Ahmadi-Khatir A (2010) Literature survey on fundamental issues of frequency control reserve (FCR) provision. Swiss Electric Research, Lausanne

[26] Restrepo JF, Galiana FD (2005) Unit commitment with frequency regulation constraints. IEEE Trans Power Syst 20(4): 1836-1842

[27] Mashhadi MR, Hossein Javidi M, Sadegh Ghazizadeh M (2011) The impacts of capabilities and constraints of generating units on simultaneous scheduling of energy and primary reserve. Electr Eng 93(3):117-126

[28] Thalassinakis EJ, Dialynas EN (2007) A method for optimal spinning reserve allocation in isolated power systems incorporating an improved speed governor model. IEEE Trans Power Syst 22(4):1629-1637

[29] Zhao JY, Zheng TX, Litvinov E (2015) Variable resource dispatch through do-not-exceed limit. IEEE Trans Power Syst 30(2):820-828

[30] Thatte AA, Zhang F, Xie L (2011) Frequency aware economic dispatch. In: Proceedings of the North American power symposium (NAPS'11), Boston, 4-6 Aug 2011, 7 pp

[31] Güler T, Gross G (2007) Detection of island formation and identification of causal factors under multiple line outages. IEEE Trans Power Syst 22(2):505-513

[32] Reid GF, Hasdorff L (1973) Economic dispatch using quadratic programming. IEEE Trans Power Appar Syst 92(6):2015-2025

[33] Sherali HD, Alameddine A (1992) A new reformulation-linearization technique for bilinear programming problems. J Global Optimiz 2(4):379-410

[34] Luo ZQ, Pang JS, Ralph D (2008) Mathematical programs with equilibrium constraints. Cambridge University Press, Cambridge

[35] Rosenthal RE (2009) GAMS-the solver manuals. General algebraic modeling system (GAMA). Development Corporation, Washington, DC, pp 131-176

[36] Zhao Q, Zhang L, Wang Q et al (2011) Impact of load frequency characteristics on frequency stability of power systems. Power Syst Technol 35(3):69-73 (in Chinese)
[37] Zimmerman RD, Murillo-Sánchez CE, Gan DD (1997) MATPOWER: a MATLAB power system simulation package. http:// www.pserc.cornell.edu/matpower/

Donglei SUN received the B.Eng. degree in electrical engineering from Shandong University, Jinan, China, in 2010. He is currently pursuing his Ph.D degree in electrical engineering from Shandong University. His research interests are power system synergistic dispatch with intermittency and renewable energy, and power system synergistic dispatch of power source and electric network.

Xueshan HAN received the B.Eng. and M.Eng. degrees from Northeast China Institute of Electric Power Engineering, Jilin, China, in 1984 and 1989, respectively, and the Ph.D degree from Harbin Institute of Technology, Harbin, China, in 1994, all in electrical engineering. From 1998 to 2000, he was a research fellow in Nanyang Technological University. He is now a Professor and serving as Vice Dean of the School of Electrical Engineering, Shandong University, Jinan, China. His research interests include energy system forecasting, power system economic dispatch, electricity market design, power system operation optimization and control.

Bo ZHANG received the B.Eng. and M.Eng. degrees in electrical engineering from Shandong Institute of Technology, Jinan, China, in 1984 and 1989, respectively. He is now a professor with the School of Electrical Engineering, Shandong University, Jinan, China. His research interests include power system dispatching with intermittency and renewable energy, power system operation and control.

Mingqiang WANG received the B.Eng. and M.Eng. degrees from Shandong University, Jinan, China, in 2004 and 2007, respectively, and the Ph.D degree from Nanyang Technological University, Singapore, in 2012, all in electrical engineering. From 2012 to 2013, he was a research fellow in Nanyang Technological University. $\mathrm{He}$ is now a lecturer with the School of Electrical Engineering, Shandong University, Jinan, China. His research interests are power system operation, economics and computation.

Tianyu DING received the B.Eng. degree in electrical engineering from Shandong University, Jinan, China, in 2010. He is currently pursuing his Ph.D degree in electrical engineering from the University of Alberta, Edmonton, AB, Canada. His research interests are power system operation optimization and control. 